\title{
FUNCIONES DISCRIMINANTES PARA ESTIMAR SEXO A PARTIR DE HUESOS LARGOS EN POBLACIONES COLONIALES DEL CENTRO OESTE DE ARGENTINA
}

\author{
DISCRIMINATING FUNCTIONS TO ESTIMATE SEX FROM LONG BONES IN \\ COLONIAL POPULATIONS OF THE CENTRAL WEST OF ARGENTINA
}

\author{
Daniela Alit Mansegosa ${ }^{1,2}$, Pablo Sebastián Giannotti ${ }^{1,2}$, Horacio Chiavazza ${ }^{3}$ y \\ Gustavo Barrientos ${ }^{1,4}$
}

\begin{abstract}
Los esqueletos incompletos y huesos humanos aislados constituyen un volumen importante del material recuperado en templos coloniales americanos. Esta situación lleva a la necesidad de realizar procedimientos que permitan la asignación sexual de cada elemento con el fin de profundizar diversos tipos de estudios bioantropológicos. El objetivo de este trabajo es desarrollar funciones discriminantes para estimar el sexo a partir de huesos largos en una muestra recuperada en tres templos coloniales de Mendoza (Argentina) correspondiente al período entre los siglos XVII y XIX. La muestra comprende 61 individuos adultos (entierros primarios completos), con sexo determinado a partir de indicadores pélvicos y craneanos. En cada caso, se tomaron un conjunto de medidas del húmero, radio, ulna, clavícula, tibia y fémur que fueron empleadas para generar funciones discriminantes. Las funciones desarrolladas permitieron estimar el sexo con un elevado porcentaje de asignaciones correctas, siendo el fémur $(92,1 \%)$ y el húmero $(90 \%)$ los elementos con porcentajes promedio de estimaciones correctas más altos y con porcentajes más elevados en el sexo masculino que en el femenino. La ulna $(79,3 \%)$, la clavícula $(79,2 \%)$, la tibia $(75,9 \%)$ y el radio $(73,3 \%)$ obtuvieron un poder de clasificación más bajo. Los resultados son discutidos considerando factores genéticos, ambientales y tafonómicos de la muestra estudiada.

Palabras claves: estimación sexual, esqueleto postcraneal, poblaciones históricas, norte de Mendoza.
\end{abstract}

Incomplete skeletons and commingled human bones constitute a significant volume of recovered material in American colonial temples. This poses the need to perform procedures that allow the sexual assignment of each element in order to deepen various types of bioanthropological studies. The objective of this work is to develop discriminant functions to estimate sex from long bones in a sample recovered in three colonial temples of Mendoza (Argentina) from the 17-18TH centuries. The sample contains 61 adult individuals (complete primary burials) with sex determined from pelvic and cranial indicators. In each case, a set of measurements of the humerus, radius, ulna, clavicle, tibia and femur was taken to generate discriminant functions. The functions thus developed allowed to estimate the sex with a high degree of reliability. The femur (92.1\%) and the humerus $(90 \%)$ were the elements with higher average percentages of correct estimates, and with higher percentages in males than in females. The ulna (79.3\%), clavicle (79.2\%), tibia (75.9\%) and radius (73.3\%) obtained a lower classification ability. The results are discussed considering the genetic, environmental, and taphonomic factors of the studied sample.

Key words: Sexual estimation, postcranial skeleton, historical populations, north of Mendoza.

Estimar el sexo a partir de huesos largos ha resultado de suma utilidad en muestras donde los indicadores clásicos, como la pelvis y el cráneo, se encuentran mal conservados, son fragmentarios o están ausentes (Black 1978; Mac Laughlin y Bruce 1985; Sakaue 2004; Šlaus 1997; Trancho et al. 1997). Asimismo, en muestras procedentes de entierros secundarios u osarios, donde es necesario conocer el sexo de los huesos largos aislados para realizar reensamblajes de individuos (Byrd 2008), para hacer cálculos de número mínimo de individuos (Ubelaker 1974) y el número más probable de individuos (Adams y Konisberg 2004). En todos los casos, poder definir el sexo en la mayor cantidad de elementos anatómicos permite ajustar los resultados de forma más precisa.

Los huesos largos presentan diferencias en el tamaño y robustez que resultan útiles para evaluar el sexo en los individuos. La estimación sexual de los restos óseos humanos constituye uno de los primeros pasos necesarios para abordar problemas paleodemográficos, patológicos, de biodistancia, entre otros (Meindl et al. 1985). El dimorfismo sexual es el resultado fundamentalmente de la acción conjunta de factores genéticos y fisiológicos, aunque también pueden intervenir factores ambientales ligados al crecimiento y desarrollo (Loth y Henneberg 2001; Oyhenart et al. 1999; Pucciarelli et al. 1993). Sibien

\footnotetext{
Consejo Nacional de Investigaciones Científicas y Técnicas (CONICET). Buenos Aires, Argentina.

2 Instituto de Arqueología y Etnología de la Facultad de Filosofía y Letras de la Universidad Nacional de Cuyo. Parque General San Martín, Mendoza, Argentina. mansegosad@yahoo.com.ar; pablosebastiangiannotti@gmail.com

3 Instituto de Arqueología y Etnología de la Facultad de Filosofía y Letras de la Universidad Nacional de Cuyo. Centro de Investigaciones Ruinas de San Francisco. Parque General San Martín, Mendoza, Argentina. hchiavazza@gmail.com

4 División Antropología, Facultad de Ciencias Naturales y Museo, Universidad Nacional de La Plata. La Plata, Argentina. barrient@museo.fcnym.unlp.edu.ar
} 
el proceso de diferenciación sexual se da desde la gestación, estas diferencias se acentúan durante la adolescencia y adultez debido a la acción de procesos hormonales (Saunders 2000).

Está demostrado que los huesos largos de los individuos masculinos, al igual que el cráneo y los dientes, presentan parámetros que dan cuenta de un mayor tamaño y robustez que los hallados en individuos femeninos de la misma población (White y Folkens 2005). El problema se centra principalmente en que la magnitud de dimorfismo sexual varía entre poblaciones, lo que conduce a un solapamiento entre valores masculinos y femeninos (Frayer y Wolpoff 1985). De esta manera, surge la necesidad de elaborar modelos regionales específicos en un intento de mejorar la exactitud en la determinación del sexo. Además, la mayoría de las muestras óseas empleadas para desarrollar criterios de estimación sexual a partir de huesos largos han sido obtenidas de poblaciones asignadas cronológicamente entre los siglos XV y XX y son principalmente de ancestría europea, africana o asiática (Asala 2001; Di Bennardo y Taylor 1982; King et al. 1998; Mall et al. 2000; Purkait y Chandra 2004; Sakaue 2004; Šlaus y Tomičić 2005). Si bien estos criterios podrían llegar a ser útiles en muestras de poblaciones históricas, en muchos casos tenemos el problema de no conocer la ancestría de los individuos analizados.

En Argentina se han aplicado análisis discriminante en huesos largos para estimar el sexo solamente en muestras prehispánicas correspondientes de la región Pampeana y Patagónica. En el caso del trabajo de Béguelin y González (2008), las funciones fueron generadas para el fémur y en Béguelin et al. (2011) para el húmero, ambos basados en muestras de Pampa y Patagonia. Por otra parte, Mazza y Béguelin (2013) aplicaron los mismos métodos en distintos huesos largos para muestras del Paraná inferior. En todos los casos, los resultados mostraron altos porcentajes de clasificaciones correctas en la asignación sexual. En nuestro caso de estudio, resulta imprescindible disponer de parámetros para clasificar los huesos largos según el sexo, ya que un gran volumen de las muestras recuperadas procede de entierros secundarios y osarios de templos coloniales ubicados en el Área Fundacional de Mendoza. Debido a las prácticas de inhumación coloniales, la remoción antrópica efectuada con el fin de liberar espacio para nuevas inhumaciones, generó un gran volumen de huesos desarticulados y mezclados, así como también esqueletos incompletos (Chiavazza 2005; Chiavazza et al. 2015; Giannotti 2016; Mansegosa 2010; Mansegosa 2010; Mansegosa y Chiavazza 2010). Adicionalmente, las poblaciones bajo estudio se conformaron mediante un proceso de mestizaje que incluyó poblaciones nativas, europeas y africanas, por esta razón es necesario evaluar el dimorfismo con modelos locales. El objetivo de este trabajo es desarrollar funciones discriminantes para poder realizar estimaciones de sexo en huesos largos de la población bajo estudio y de esta manera mejorar la estimación sexual de las muestras y avanzar en el conocimiento de su estructura demográfica.

\section{Materiales y Métodos}

La muestra seleccionada para este estudio procede de tres templos coloniales: Ruinas de San Francisco (RSF), La Caridad (LC) y Santo Domingo (SD), ubicados en el Área Fundacional de Mendoza, Argentina como se demuestra en la Figura 1. El rango cronológico de los templos abarca los siglos XVII y XIX (Chiavazza et al. 2015). Los mismos han sido excavados en varias etapas entre los años 1995 y 2012 por el Centro de Investigaciones Ruinas de San Francisco. Como se observa en la Tabla 1, la muestra está compuesta por 61 individuos adultos, de los cuales se pudo estimar el sexo en 52 casos, 23 resultaron masculinos y 29 femeninos. La determinación del sexo se realizó a partir de estructuras diagnósticas reconocidas. En la pelvis, el arco ventral, la concavidad subpúbica, la rama isquiopúbica (Phenice 1969 en Buikstra y Ubelaker 1994) y el ángulo de la escotadura ciática mayor. En el cráneo, la proyección de la cresta nucal, el tamaño del proceso mastoides, el espesor del margen supraorbital y la proyección de la eminencia mentoniana (Buikstra y Ubelaker 1994). En los 9 individuos restantes no se pudo determinar el sexo debido a que no tienen dichas estructuras diagnósticas por encontrarse en mal estado de conservación o por estar ausentes por procesos de remoción antrópicos postdepositacionales (Chiavazza 2005). La edad al momento de la muerte se estimó de acuerdo con los cambios morfológicos de la sínfisis púbica (Brooks y Suchey 1990; Todd 1921) y de la superficie auricular (Lovejoy et al. 1985).

La información etnohistórica indica que en estos templos se inhumaron a personas de diferente ascendencia biológica (españoles, nativos, africanos y mestizos) (Bárcena y Mulle 2011; Mansegosa 2015a, 2015b). Investigaciones previas sobre la variación morfológica de las muestras aquí estudiadas indican que, si bien hay diferencias en la estatura y en la platimería de los individuos a nivel cronológico y entre los lugares de entierro, éstas no son significativas desde el punto de vista estadístico (Mansegosa 2015, 2016a). Por esta razón, consideramos que la muestra utilizada es adecuada para realizar funciones discriminantes a pesar de la variación poblacional, propia de las colonias españolas americanas. Para una descripción más detallada de las muestras ver Chiavazza (2005) y Mansegosa $(2015,2016 b)$.

Se relevaron 35 variables métricas del húmero, radio, ulna, clavícula, tibia y fémur, seleccionadas entre las variables estándar más utilizadas (Buikstra y Ubelaker 1994). El instrumental utilizado para la medición fue tabla osteométrica para medir longitudes y anchos, cinta métrica para las circunferencias y un calibre digital (Baker, precisión $0,01 \mathrm{~mm}$ ) para los diámetros. Se priorizaron los elementos de lateralidad izquierda pero, cuando no estaba disponible, se reemplazó por los del lado derecho. En la Tabla 2 se detallan cada una de las variables relevadas, su abreviatura y el instrumental de medición empleado.

La muestra presenta grados de preservación heterogéneos debido a procesos postdepositacionales 


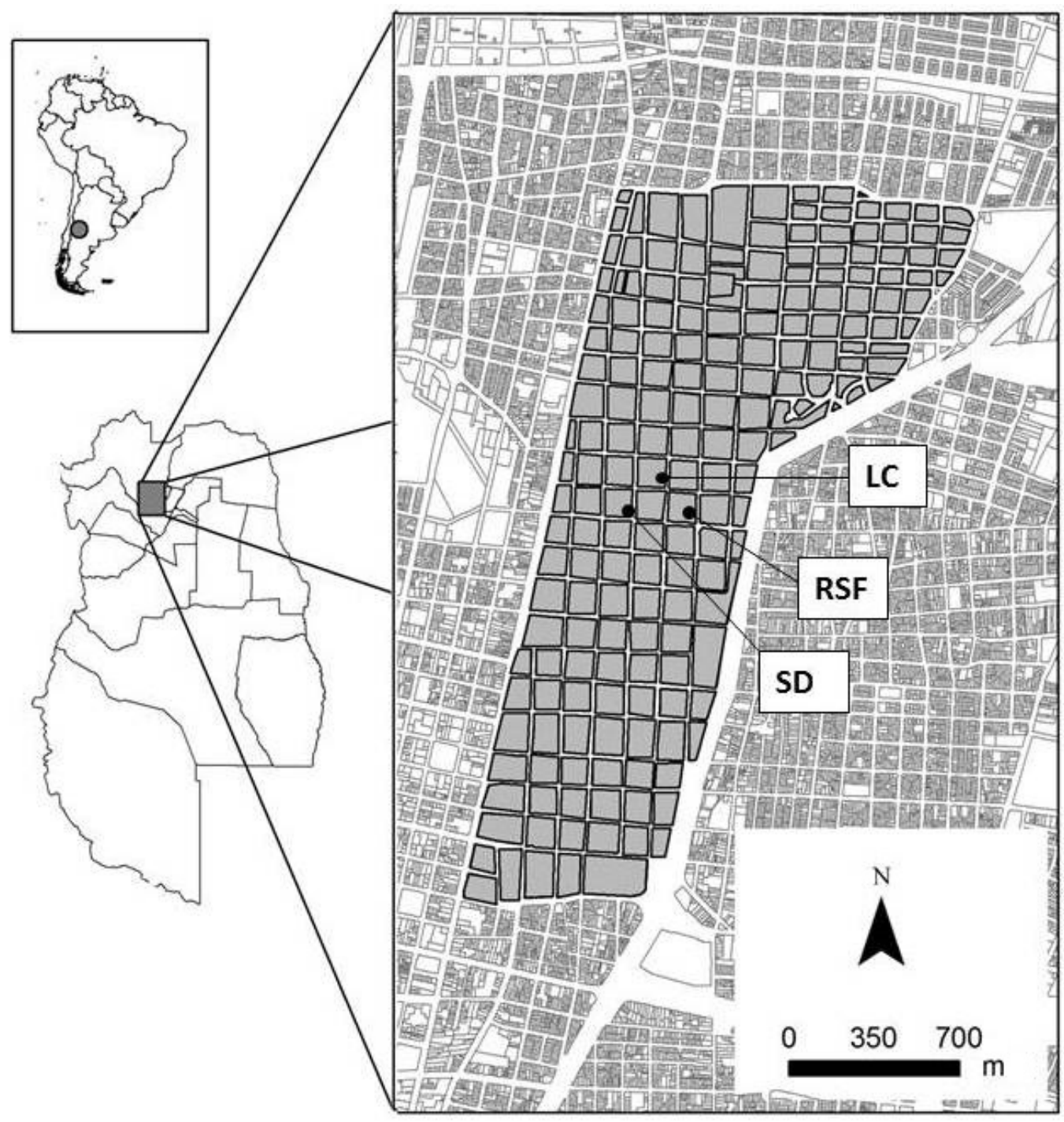

Figura 1. Procedencia geográfica de la muestra analizada. Se indica la procedencia de los puntos arqueológicos: LC (La Caridad), RSF (Ruinas de San Francisco) y SD (Santo Domingo). Los mismos se ubican dentro del Área Fundacional de la antigua ciudad de Mendoza (Argentina).

Geographic origin of the sample analyzed. Localization of archaeological points: LC (La Caridad), RSF (Ruins of San Francisco) and SD (Santo Domingo). They are located within the Foundational Area of the old city of Mendoza (Argentina).

Tabla 1. Cantidad de individuos analizados según sexo y punto arqueológico.

Number of individuals analyzed according to sex and archaeological point.

\begin{tabular}{ccccc}
\hline $\begin{array}{c}\text { Punto } \\
\text { Arqueológico }\end{array}$ & Masculinos & $\begin{array}{c}\text { Sexo } \\
\text { Femeninos }\end{array}$ & Indeterminados & Total \\
\hline LC & 12 & 8 & 2 & 22 \\
RSF & 11 & 21 & 5 & 37 \\
SD & - & - & 2 & 2 \\
Total & 23 & 29 & 9 & 61 \\
\hline
\end{tabular}


Tabla 2. Variables analizadas. TO: tabla osteométrica; C: calibre digital; CM: cinta métrica. Variables analyzed. TO: osteometric table; $C$ : digital caliber; CM: tape measure.

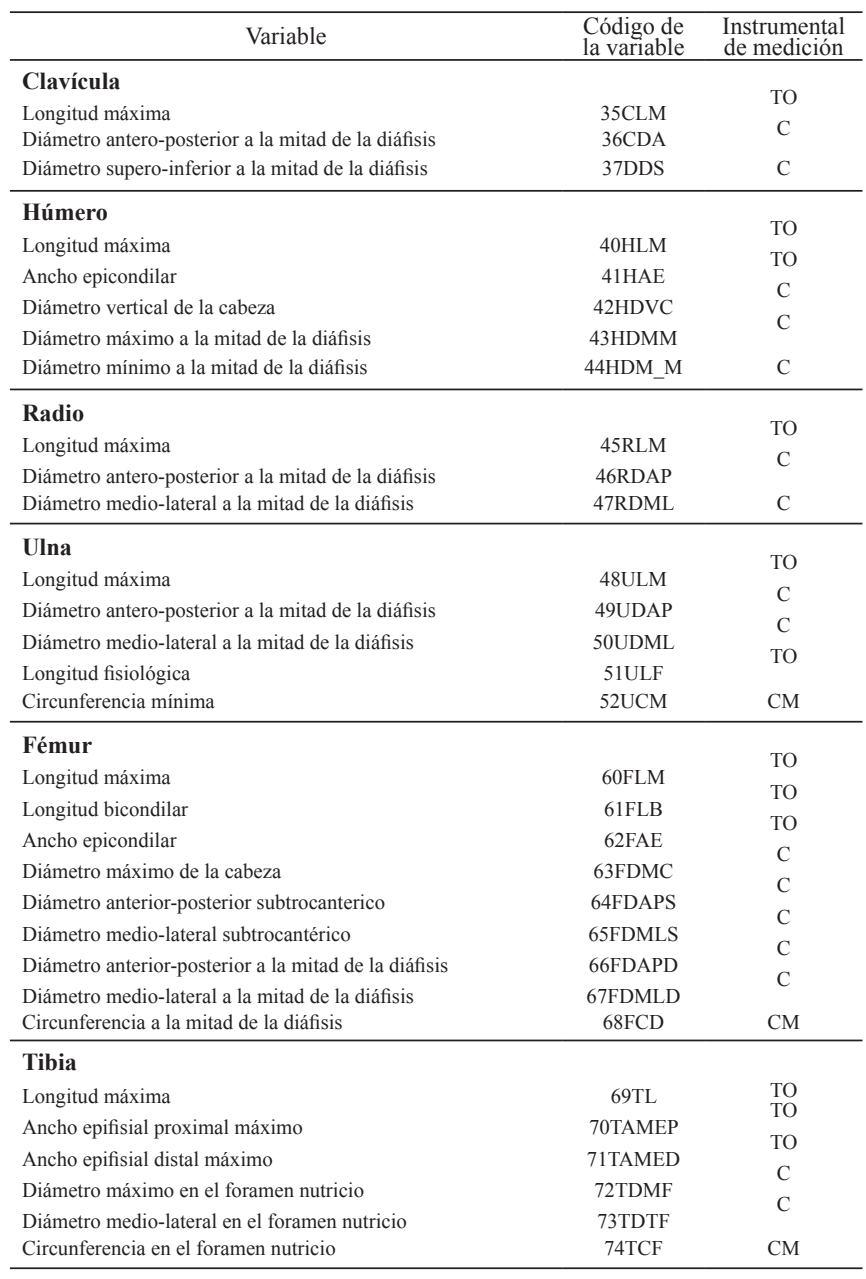

naturales y antrópicos. Por esta razón algunas variables no se pudieron medir, principalmente las que implican la región de las epífisis, ya que es en este sector donde se observa la mayor pérdida de material (Mansegosa 2015). Esto generó la pérdida de valores correspondientes a las variables que registran las dimensiones de esa estructura. Tales valores perdidos fueron simulados a partir de valores presentes en otras variables mediante el método de imputación múltiple con la opción EM algorithm (Schafer y Graham 2002; Schafer y Olsen 1998). Previo a la imputación, se controló que ninguna de las matrices tuviera una alta proporción de datos perdidos ya que el método es suficientemente robusto con porcentajes de datos perdidos hasta del 35 o $40 \%$ (Schafer y Olsen 1998). De todos los elementos óseos relevados la tibia presentó la mayor cantidad de valores perdidos ascendiendo a $35 \%$. A partir de las nuevas matrices se verificó la distribución normal de las variables mediante el estadístico ShapiroWilk $(\mathrm{p}<0,05)$ y se calcularon medias y desvíos estándar de cada una de ellas. Asimismo, se evaluó la presencia de diferencias significativas a entre los sexos mediante el empleo de una prueba de diferencia de medias para muestras independientes.

Luego se procedió con el análisis discriminante y la elaboración de su fórmula. El análisis discriminante consiste en la identificación de variables que diferencian (discriminan) a dos o más grupos, y en base a las cuales se crea una función capaz de discriminar con la mayor precisión posible a los individuos de uno u otro grupo (Hair et al. 1999). En este trabajo, la incorporación de la variable en la fórmula se llevó a cabo a través del método stepwise forward (paso a paso), el cual selecciona y añade al modelo las variables que más discriminan entre ambos grupos (en este caso sexo masculino y femenino). Cuando la incorporación de una nueva variable no aumenta la eficiencia para separar los grupos de manera significativa, concluye el análisis (Hair et al. 1999).

Con el fin de establecer la confiabilidad y capacidad de predicción de las funciones generadas, se utilizó el método de validación cruzada. Este método consiste en la creación de una nueva función con todos los individuos menos uno (el que se va a clasificar) y se evalúa su correcta asignación 
al grupo de pertenencia (femenino o masculino). El procedimiento se repite con todos los casos para obtener un porcentaje de clasificación correcta que resulta en una buena valoración del modelo. En todos los análisis se utilizó el paquete estadístico SPSS Statistics v.19. Con el objetivo de evaluar la utilidad del modelo generado en este trabajo, se estimó el sexo de los individuos analizados mediante otras funciones obtenidas en poblaciones de otras regiones y cronologías: prehispánicas de Argentina (Béguelin y González 2008; Béguelin et al. 2010; Mazza y Béguelin 2013), españolas y anglosajonas de la Edad Media (siglos V al XI) (Pomeroy y Zakrzewski 2009) y poblaciones negras del sur de África (Asala 2001; Barrier y L'Abbé 2008).

Finalmente, se aplicaron las fórmulas discriminantes generadas con el objetivo de estimar el sexo en los 9 individuos indeterminados, procedentes de la región de estudio.

\section{Resultados}

En términos generales el análisis de las variables métricas relevadas, muestra un predominio de elementos anatómicos representados por individuos femeninos (promedio=19) por sobre los masculinos (promedio=13). También se observa un mayor tamaño promedio de los masculinos por sobre los femeninos, donde la prueba de $t$ para muestras independientes arrojó diferencias significativas $(p<0,05)$ para todas las variables. En la Tabla 3 se resumen las estadísticas univariadas para todas las variables por elemento anatómico y por sexo, y la cantidad de elementos observados.

A partir de las variables relevadas, el método stepwise forward (paso a paso) seleccionó aquellas que mostraban mayor discriminación entre los sexos. En la Tabla 4 se presentan dichas variables y sus estadísticos para derivar la función discriminante. De cada variable se especifica el valor de lambda de Wilks ( $\lambda$ ) y de F. El valor de lambda de Wilks varía entre cero y uno, cuanto más cercano a cero es su valor y mayor es el valor de F, mayor es la capacidad de discriminación que tiene la variable. El fémur muestra la capacidad discriminatoria más alta en relación con los demás elementos $(\lambda=0,367)$. Le sigue el húmero con dos variables $(\lambda=0,49)$. La ulna y la tibia tienen el mismo valor de $\lambda(0,556)$ lo que indica un ajuste moderado. Por el contrario, el radio y la clavícula presentan los valores más

Tabla 3. Variables analizadas, media y desvío estándar de cada una y cantidad de elementos observados $(\mathrm{N})$. Variables analyzed; mean and standard deviation of each one; and number of observed elements $(N)$.

\begin{tabular}{|c|c|c|c|c|c|c|c|}
\hline \multirow{2}{*}{ Elemento } & \multirow{2}{*}{ Variable } & \multicolumn{3}{|c|}{ Masculinos } & \multicolumn{3}{|c|}{ Femeninos } \\
\hline & & $\mathrm{N}$ & Media & Desvío estándar & $\mathrm{N}$ & Media & Desvío estándar \\
\hline \multirow{3}{*}{ Clavícula } & & 8 & & & 16 & & \\
\hline & $35 \mathrm{CLM}$ & & 145,3 & 8,22 & & 138,6 & 6,66 \\
\hline & $\begin{array}{l}36 \mathrm{CDA} \\
\text { 37DDS }\end{array}$ & & $\begin{array}{c}14,04 \\
11\end{array}$ & $\begin{array}{l}2,12 \\
1,67\end{array}$ & & $\begin{array}{c}11,64 \\
9,57\end{array}$ & $\begin{array}{l}1,45 \\
1,29\end{array}$ \\
\hline \multirow{5}{*}{ Húmero } & & 17 & & & 23 & & \\
\hline & 40HLM & & 313,42 & 12,86 & & 293,70 & 8,29 \\
\hline & 41HAE & & 60,38 & 3,47 & & 55,69 & 4,43 \\
\hline & $42 \mathrm{HDVC}$ & & 44,85 & 3,17 & & 37,98 & 6,21 \\
\hline & $\begin{array}{l}\text { 43HDMM } \\
\text { 44HDM_M }\end{array}$ & & $\begin{array}{l}24,11 \\
19,81\end{array}$ & $\begin{array}{l}2,49 \\
2,34\end{array}$ & & $\begin{array}{l}20,71 \\
16,02\end{array}$ & $\begin{array}{l}2,47 \\
1,94\end{array}$ \\
\hline \multirow{3}{*}{ Radio } & & 13 & & & 17 & & \\
\hline & 45RLM & & 224,16 & 5,01 & & 218,13 & 11,08 \\
\hline & $\begin{array}{l}\text { 46RDAP } \\
\text { 47RDML }\end{array}$ & & $\begin{array}{l}13,68 \\
15,95 \\
\end{array}$ & $\begin{array}{l}2,25 \\
2,72 \\
\end{array}$ & & $\begin{array}{c}11,1 \\
14,34 \\
\end{array}$ & $\begin{array}{l}1,35 \\
1,91\end{array}$ \\
\hline \multirow{5}{*}{ Ulna } & & 11 & & & 18 & & \\
\hline & 48ULM & & 276,98 & 34,81 & & 234,44 & 10,98 \\
\hline & 49UDAP & & 15,77 & 3,01 & & 13,3 & 2,03 \\
\hline & 50UDML & & 15,09 & 2,6 & & 11,9 & 1,64 \\
\hline & $\begin{array}{c}51 \mathrm{ULF} \\
52 \mathrm{UCM}\end{array}$ & & $\begin{array}{c}245,83 \\
43,09\end{array}$ & $\begin{array}{c}29,95 \\
4,93\end{array}$ & & $\begin{array}{c}208,48 \\
36,07\end{array}$ & $\begin{array}{c}16,02 \\
3,22\end{array}$ \\
\hline \multirow{9}{*}{ Fémur } & & 18 & & & 20 & & \\
\hline & $60 \mathrm{FLM}$ & & 446,99 & 9,98 & & 414,03 & 17,46 \\
\hline & 61FLB & & 443,51 & 10,33 & & 408,86 & 18,4 \\
\hline & $62 \mathrm{FAE}$ & & 83,85 & 3,33 & & 74,86 & 6,94 \\
\hline & 63FDMC & & 47,32 & 2,23 & & 42,66 & 3,44 \\
\hline & 64FDAPS & & 26,68 & 1,82 & & 23,78 & 2,37 \\
\hline & 65FDMLS & & 33,48 & 2,19 & & 29,85 & 3,11 \\
\hline & 66FDAPD & & 30,89 & 2,44 & & 26,06 & 2,43 \\
\hline & $\begin{array}{c}\text { 67FDMLD } \\
\text { 68FCD }\end{array}$ & & $\begin{array}{c}29,2 \\
97,01\end{array}$ & $\begin{array}{l}2,32 \\
6,34\end{array}$ & & $\begin{array}{l}25,68 \\
84,78\end{array}$ & $\begin{array}{c}2 \\
6,54\end{array}$ \\
\hline \multirow{5}{*}{ Tibia } & & 11 & & & 18 & & \\
\hline & 69TL & & 369,04 & 42,86 & & 339,05 & 17,59 \\
\hline & $\begin{array}{l}\text { 70TAMEP } \\
\text { 71TAMED }\end{array}$ & & $\begin{array}{l}74,48 \\
34,69\end{array}$ & $\begin{array}{c}5,2 \\
4,71\end{array}$ & & $\begin{array}{l}68,97 \\
46,92\end{array}$ & $\begin{array}{l}7,98 \\
3,18\end{array}$ \\
\hline & 72TDMF & & 27,26 & 5,17 & & 31,04 & 3,12 \\
\hline & $\begin{array}{l}\text { 73TDTF } \\
\text { 74TCF }\end{array}$ & & $\begin{array}{l}27,26 \\
100,2\end{array}$ & $\begin{array}{c}5,17 \\
10,13\end{array}$ & & $\begin{array}{l}21,14 \\
85,99\end{array}$ & $\begin{array}{l}2,56 \\
6,43\end{array}$ \\
\hline
\end{tabular}


Tabla 4. Análisis discriminante de cada elemento anatómico y función derivada. Discriminant analysis of each anatomical element and derived function.

\begin{tabular}{|c|c|c|c|c|c|c|}
\hline $\begin{array}{l}\text { Elemento } \\
\text { anatómico }\end{array}$ & $\begin{array}{c}\text { Variable } \\
\text { seleccionada }\end{array}$ & Coeficiente & Constante & $\begin{array}{l}\text { Lambda } \\
\text { de Wilks }\end{array}$ & $\mathrm{F}$ & Función Discriminante \\
\hline Clavícula & $36 \mathrm{CDA}$ & 0,590 & $-7,340$ & 0,672 & 10,73 & $\left(36 \mathrm{CDA}^{*} 0,590\right)-7,340$ \\
\hline \multirow{2}{*}{ Húmero } & 42HDVC & 0,091 & \multirow{2}{*}{$-10,164$} & 0,490 & 19,235 & \multirow{2}{*}{$\left(42 \mathrm{HDVC}^{*} 0,091\right)+\left(44 \mathrm{HDM} \mathrm{M}^{*} 0,364\right)-10,16$} \\
\hline & 44HDM_M & 0,364 & & 0,549 & 31,248 & \\
\hline Radio & 46RDAP & 0,557 & $-6,807$ & 0,648 & 15,206 & $(46 \mathrm{RDAP} * 0,557)-6,807$ \\
\hline Ulna & $52 \mathrm{UCM}$ & 0,253 & $-9,817$ & 0,556 & 21,603 & $(52 \mathrm{UCM} * 0,253)-9,817$ \\
\hline \multirow{2}{*}{ Fémur } & $61 \mathrm{FLB}$ & 0,476 & \multirow{2}{*}{$-27,003$} & 0,420 & 49,652 & \multirow{2}{*}{$\left(61 \mathrm{FLB}^{*} 0,476\right)+\left(68 \mathrm{FCM}^{*} 0,745\right)-27,003$} \\
\hline & $68 \mathrm{FCM}$ & 0,745 & & 0,367 & 30,226 & \\
\hline Tibia & 74TCF & 0,124 & $-11,418$ & 0,556 & 21,534 & $\left(74 \mathrm{TCF}^{*} 0,124\right)-11,418$ \\
\hline
\end{tabular}

elevados de $\lambda$ ( 0,648 y 0,672 respectivamente), por lo que sus funciones tienen una capacidad relativamente menor de estimación del sexo. En todos los casos el valor de F es alto (valor crítico mayor a 3,84). Por otra parte, todos los modelos resultaron significativos ( $\mathrm{p}<0,001)$, lo que indica que todas las variables seleccionadas discriminan.

Tanto en el fémur como en el húmero, se seleccionaron dos variables de análisis para construir el modelo. En el resto de los elementos seleccionó una única variable. En la clavícula, húmero, radio, fémur se seleccionaron variables relacionadas al diámetro de las diáfisis. Además en el caso del húmero se seleccionó el diámetro de la epífisis proximal y en el fémur la longitud bicondilar. Mientras que en la ulna y tibia las variables utilizadas corresponden a los perímetros de la diáfisis.

En la última columna de la Tabla 4 se presenta la función discriminante correspondiente a cada elemento, que puede ser empleada para la estimación del sexo de nuevos individuos correspondientes al período histórico de la región. Los valores que resulten mayores a cero (positivos) indican una clasificación dentro del grupo de sexo masculino y los valores menores a cero (negativos) se clasifican como femeninos.

En la Tabla 5 se muestran los resultados de la aplicación del método de validación cruzada y los de las funciones de otras poblaciones. Los resultados del modelo generado en este trabajo indican frecuencias elevadas de clasificaciones correctas, con promedios para los dos sexos que varían entre $73,3 \%$ y $92,1 \%$. El fémur y el húmero presentan la mayor cantidad de casos asignados correctamente $(92,1 \%$ y $90 \%$, respectivamente), con altos porcentajes en ambos sexos. El poder de discriminación de la función del radio muestra la menor cantidad de asignaciones correctas en promedio $(73,3 \%)$, resultado esperable considerando los valores del análisis discriminante. Contrariamente, la aplicación de funciones discriminantes de otras poblaciones, en general, muestran porcentajes bajos de asignaciones correctas, principalmente en los individuos masculinos. Sólo las funciones para húmero y fémur de los españoles arrojaron elevados porcentajes para ambos sexos (entre 69,2 y 100\%).
A continuación, se estimó el sexo utilizando las funciones discriminantes propuestas en nueve individuos provenientes de RSF, LC y SD. Los mismos no poseían pelvis ni cráneo ya sea por problemas de preservación o de remoción antrópica. Es importante aclarar que en los individuos LC19 y SFNC7, si bien se encontraban presentes algunos huesos del postcráneo, no se preservó la variable utilizada por el modelo, por lo que continuaron siendo indeterminados. En los siete restantes sí se pudieron calcular algunas de las funciones, resultando cuatro masculinos, dos femeninos y uno indeterminado.

\section{Discusión y Conclusión}

Los resultados de las funciones discriminantes desarrolladas a partir de los huesos largos permitieron estimar el sexo con un elevado porcentaje de asignaciones correctas. Los porcentajes promedio de estimaciones correctas más altos se hallaron en el fémur $(92,1 \%)$ y en el húmero (90\%). Estos resultados son coincidentes con estudios realizados en otras poblaciones donde el fémur y el húmero presentan un elevado grado de dimorfismo y por lo tanto un elevado poder de clasificación (Bass 1995; Barnes y Wescott 2008; Béguelin et al. 2011; Byers 2002; İşcan et al. 1998; Robinson y Bidmos 2009; Steyn y İşcan 1999). En el caso del fémur, el 100\% de los masculinos fueron clasificados correctamente mientras que en femeninos el 85\%. En el húmero también se observa esta tendencia, con mayores porcentajes de clasificaciones correctas masculinas (94\%) que femeninas (87\%). Las diferencias en la precisión de las clasificaciones correctas entre sexos puede ser resultado de una serie de factores tales como la variación en el tamaño de la muestra y la variabilidad intra sexo. Por otro lado, en la ulna se alcanzó una precisión de $79,3 \%$ y en el radio sólo de $61,5 \%$, siendo este último elemento el que presentó el poder de clasificación más bajo. Otros investigadores también han observado que los huesos del antebrazo suelen tener moderado poder de discriminación y que sus variables correspondientes a diferentes dimensiones transversales de la diáfisis son las que mejor describen el dimorfismo (Cowal y Pastor 2008; 
Tabla 5. Porcentajes de clasificaciones correctas obtenidas con las funciones discriminantes de las muestras utilizadas en este trabajo (por validación cruzada en negritas) y de otras muestras (utilizando funciones publicadas). $\mathrm{F}=$ femenino, $\mathrm{M}=$ masculino; vnr= variable no relevada (la fórmula requiere una variable no relevada en este estudio); se colocó un guion (-) en los casos donde no hay funciones discriminantes disponibles. Percentages of correct classifications with the discriminant functions of the samples used in this work (by cross-validation in bold) and of other signals $F=$ female, $M=$ male; $v n r=$ variable not surveyed (the formula requires a variable not surveyed in this study); (-)= there are no discriminant functions available.

\begin{tabular}{|c|c|c|c|c|c|c|c|c|c|c|c|c|c|c|c|c|c|c|}
\hline & \multicolumn{3}{|c|}{ Clavícula } & \multicolumn{3}{|c|}{ Húmero } & \multicolumn{3}{|c|}{ Radio } & \multicolumn{3}{|c|}{ Ulna } & \multicolumn{3}{|c|}{ Fémur } & \multicolumn{3}{|c|}{ Tibia } \\
\hline & M & $\mathrm{F}$ & $\mathrm{T}$ & M & $\mathrm{F}$ & $\mathrm{T}$ & M & $\mathrm{F}$ & $\mathrm{T}$ & M & $\mathrm{F}$ & $\mathrm{T}$ & $\mathrm{M}$ & $\mathrm{F}$ & $\mathrm{T}$ & M & $\mathrm{F}$ & $\mathrm{T}$ \\
\hline Centro-Oeste. XVII-XIX. & 87,5 & 75 & 79,2 & 94 & 87 & 90 & 61,5 & 82,4 & 73,3 & 81,8 & 77,8 & 79,3 & 100 & 85 & 92,1 & 72,7 & 77,8 & 75,9 \\
\hline $\begin{array}{l}\text { Norte de Patagonia-Sur Pampa } \\
\text { (Béguelin y González 2008; } \\
\text { Béguelin et al. 2010) }\end{array}$ & - & - & - & 55,5 & 100 & 76 & - & - & - & - & - & - & 0 & 100 & 50 & - & - & - \\
\hline
\end{tabular}

Sur Patagonia (Béguelin y

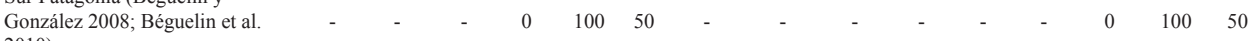
2010)

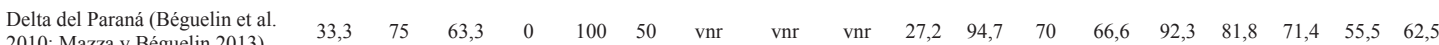

2010; Mazza y Béguelin 2013)

\begin{tabular}{|c|c|c|c|c|c|c|c|c|c|c|c|c|c|c|c|c|c|c|}
\hline $\begin{array}{l}\text { Españoles (Pomeroy y Zakrzewski } \\
\text { 2009; González-Reimers 2000) }\end{array}$ & 33,3 & 62,5 & 54,5 & 100 & 69,2 & 80 & 60 & 70 & 66,6 & 0 & 33,3 & 10,5 & 80 & 81,8 & 80,9 & 75 & 60 & 66,6 \\
\hline $\begin{array}{l}\text { Anglosajones (Pomeroy y } \\
\text { Zakrzewski 2009) }\end{array}$ & 33,3 & 75 & 63,3 & 14,3 & 92,3 & 65 & 20 & 100 & 73,3 & 50 & 92,3 & 78,9 & 40 & 100 & 71,4 & - & - & - \\
\hline $\begin{array}{l}\text { Sudáfrica (Barrier y L'Abbé 2008; } \\
\text { Asala 2001) }\end{array}$ & vnr & vnr & vnr & vnr & $\mathrm{vnr}$ & vnr & 33,3 & 90 & 68,7 & 88,8 & 25 & 52,4 & 85 & 15,8 & 51,3 & - & - & - \\
\hline
\end{tabular}

Sakaue 2004; Waghmare et al. 2012). La precisión de la clasificación de la tibia fue de $75,9 \%$, sin embargo en otras poblaciones se han obtenido resultados más favorables que oscilan entre 80 y $98 \%$ (Gonzales Reimers et al. 2000; İsçan et al. 1994; Ślaus y Tomičić 2005; entre otros). Considerando que el dimorfismo sexual en las poblaciones humanas se expresa en un mayor tamaño corporal general en los individuos masculinos (Frayer y Wolpoff 1985; Leigh 1992), es esperable que las variables lineales varíen en todos los elementos de manera coincidente como es en los elementos empleados en este trabajo.

En general, los porcentajes de clasificaciones correctas alcanzados resultan similares a los obtenidos en muestras de poblaciones prehispánicas de Argentina. En muestras de Pampa y Patagonia con distinta procedencia geográfica y temporal, se lograron estimaciones correctas para el fémur que van de 84 a 90\% (Béguelin y González 2008) y en el húmero de 85 a 97\% (Béguelin et al. 2011). En muestras del Delta del Paraná inferior (provincia de Entre Ríos), Mazza y Béguelin (2013) calcularon funciones para distintos huesos largos; la clavícula fue el elemento que mejor clasificó con $97 \%$ de precisión, seguido del húmero $96 \%$, el fémur $90 \%$, la tibia $87 \%$ y por último el radio $86 \%$, el que menor poder de clasificación presentó. Estos valores resultan un poco más altos que los alcanzados en este trabajo.

El patrón de dimorfismo observado puede deberse a la combinación de una serie de factores genéticos, ambientales y tafonómicos. Por un lado, la variabilidad poblacional que dio origen a la muestra, individuos procedentes de distintas poblaciones de origen europeo, americano y africano. Sin embargo, debido a que la mayoría de los individuos empleados en este estudio pertenecen a los siglos XVIII y XIX suponemos que pueden ser de ascendencia principalmente mestiza. Esta interpretación se basa en estudios métricos craneales y postcraneales efectuados en estas muestras que dan cuenta de poblaciones con marcada variabilidad morfológica (Mansegosa 2015a, 2015b). Asimismo, investigaciones históricas sugieren que el proceso de mestizaje (i.e. flujo génico) habría comenzado en la región desde épocas muy tempranas dado el desbalance entre los sexos en las distintas poblaciones (Comadrán Ruiz 1969; Prieto 2000; Zuluaga 1970) por lo que el alto mestizaje podría estar influyendo en el dimorfismo observado. Por otra parte, se ha propuesto que el grado de dimorfismo disminuye en poblaciones bajo procesos de desnutrición y enfermedades ya que resulta fisiológicamente costoso producir rasgos sexuales secundarios masculinos (Cameron 2002; Gimarey et al. 1996; Lieberman 1982). En las poblaciones bajo estudio se han registrado altos niveles de estrés metabólico evidenciado por hipoplasias de esmalte dental, hiperostosisis porótica y cribra orbitalia (Giannotti 2016; Mansegosa 2015, 2016; Mansegosa y Chiavazza 2010). Esto puede haber influido en el tamaño y robustez de los individuos masculinos disminuyendo el grado de dimorfismo sexual, lo que explica que muchas mujeres sean clasificadas incorrectamente como masculinos. Por último, la cantidad de datos simulados puede haber aumentado la variación y en consecuencia el patrón del dimorfismo observado, ya que algunos casos como la variable 69TL de la tibia presentó el 35\% de datos 
Tabla 6. Estimación sexual de individuos incompletos de RSF y LC. M Masculino, F: Femenino; I: indeterminados. Sexual estimation of incomplete individuals of RSF and LC. M: Male, F: Female I: undetermined.

\begin{tabular}{lccccccc}
\hline \multirow{2}{*}{ Individuo } & \multicolumn{7}{c}{ Resultado de la función discriminante por cada elemento } \\
& Clavícula & Húmero & Radio & Ulna & Fémur & Tibia & Sexo \\
\hline SFNC10 & 0,08 & - & - & - & - & - & M \\
SFNC15 & 0,01 & - & $-0,47$ & - & $-1,12$ & $-1,12$ & F \\
SFNC8 & - & - & 2,5 & 1,06 & $-0,40$ & - & I \\
SFC18 & - & - & 1,21 & - & - & - & M \\
LC2 & - & - & - & - & 0,01 & - & M \\
LC19 & - & - & - & - & - & - & I \\
SFNC7 & - & - & - & - & - & - & I \\
SD1 & - & $-0,62$ & - & - & $-0,37$ & - & F \\
SD2 & - & - & - & - & 1,59 & 0,98 & M \\
\hline
\end{tabular}

perdidos, porcentaje cercano al límite sugerido para este tipo de estudios (Schafer y Olsen 1998).

En este trabajo, el método empleado (stepwise forward) seleccionó una sola variable para la mayoría de los elementos, exceptuando el fémur y húmero, donde además de medidas de la diáfisis seleccionó la longitud bicondilar y el diámetro vertical de la cabeza, respectivamente. Las variables vinculadas a la circunferencia de la diáfisis y con el tamaño de las epífisis son buenosindicadores sexuales ya que se correlacionan con el factor tamaño (Ruff 1994), así como también con el peso y musculatura (DiBennardo y Taylor 1982; İşcan y Miller-Shaivitz 1984). Resulta llamativo que en el fémur no se seleccionó la variable diámetro máximo de cabeza, ya que es una de las estructuras más dimórficas halladas en huesos largos (Asala 2001; Mall et al. 2000; Steyn e İsçan 1997). Sin embargo, King et al. (1998) también calcularon que la longitud bicondilar es un buen indicador para el disgnóstico del sexo.

La aplicación de las fórmulas a los individuos de sexo indeterminado permitió una clasificación en la mayoría de los casos. Solo dos individuos no pudieron ser clasificados debido a que no se conservó la variable requerida para la aplicación de la función. Esto resulta un problema frecuente en registros fragmentarios como es nuestro caso de estudio con entierros secundarios y osarios. Esta situación lleva a la necesidad de elaborar funciones para cada una de las variables métricas relevadas, tal como proponen algunos autores (Šlaus 1997; Šlaus et al. 2003; Trancho et al. 1997).

Los resultados de la estimación de sexo a partir de funciones generadas en otras poblaciones fueron inexactos, lo que ratifica la importancia de generar modelos regionales para la evaluación del dimorfismo sexual. Los individuos masculinos del período colonial del centro-oeste argentino tendrían un menor tamaño que los nativos sudamericanos, africanos y anglosajones dado que las clasificaciones correctas con estos modelos resultaron muy bajas o nulas. Si bien, con dichas funciones las mujeres presentaron altos porcentajes de asignaciones correctas, éstas no resultan útiles ya que muchos masculinos pueden ser clasificados como femeninos, debido al menor tamaño corporal. Es importante destacar que las funciones para fémur y húmero de las poblaciones españolas, dieron altas asignaciones correctas para los dos sexos. Esto puede interpretarse como similares tamaños y patrones de dimorfismo de las variables analizadas en ambas muestras, la española y la de estudio. Sin embargo, en el resto de los huesos largos postcraneales las clasificaciones correctas fueron bajas. Esto sugiere que la población española podría ser más cercana biológicamente a las poblaciones coloniales estudiadas, lo que se explica a partir de la conquista europea. Sin embargo el patrón de dimorfismo varía en los huesos postcraneales y no tienen el mismo poder de clasificación entre dichas poblaciones, por lo que se sugiere utilizar fórmulas específicas de la población de estudio.

En síntesis, nuestros resultados muestran que cuando no se dispone de todo el esqueleto o de estructuras diagnósticas del sexo como la pelvis y el cráneo, las medidas de los huesos largos constituye una alternativa adecuada para estimar el sexo. Esta situación es muy frecuente en el caso de los entierros secundarios de los templos coloniales americanos, originados debido a la intensa reutilización de estos espacios confinados como cementerios. Bajo tales circunstancias es fundamental contar con técnicas de estimación sexual confiables basadas en el análisis de unidades anatómicas individuales y específicas de la población de estudio. Las funciones generadas permitieron clasificar a los individuos con alto grado de confiabilidad, a pesar de ser poblaciones con alto grado de mestizaje típico de este tipo de poblaciones. Asimismo, en futuros estudios se prevee la incorporación de análisis de ADN antiguo, lo que permitirá contar con un estimador independiente y no osteológico del sexo por amelogenina.

Agradecimientos. La investigación fue realizada en el marco de una beca postdoctoral otorgada por el CONICET (a DAM). Los miembros del Centro de Investigaciones Ruinas de San Francisco colaboraron en la excavación de las muestras empleadas. Agradecemos a los evaluadores que realizaron valiosos comentarios sobre una versión anterior de este trabajo.

\section{Referencias Citadas}

Adams, B.J. y L.W. Konisberg 2004. Estimation of the most likely number of individuals from commingled human skeletal remains. American Journal of Physical Anthropology 125:138-151.

Asala, S.A. 2001. Sex determination from the head of the femur of South African whites and blacks. Forensic Science International 117:15-22.
Bárcena, J.R. y M. Pannuzzio de Mulle 2011. Iglesia y Convento de Santo Domingo Soriano: aportes sobre el registro documental de inhumaciones y arqueológico de ocupaciones en su predio del área fundacional de Mendoza. Xama $\mathrm{N}^{\circ} 3$. Serie de Monografías. INCIHUSA CONICET, Mendoza. 
Barnes, A.J. y D.J. Wescott 2008. Sex determination of Mississippian skeletal remains from humeral measurements. Missouri Archaeologist 68:133-137.

Barrier, I.L.O. y E.N. L'Abbe 2008. Sex determination from the radius and ulna in a modern South African simple. Forensic Science International 179:85.e1-85.e7.

Bass, W.M. 1995. Human Osteology: A Laboratory and Field Manual, Missouri Archaeological Society, Columbia.

Béguelin, M., F. Lotto y P. González 2011. Estimación del sexo en cazadores-recolectores de Sudamérica a partir de variables métricas del húmero. Intersecciones en Antropología 12:61-68.

Béguelin, M. y P. González 2008. Estimación del sexo en poblaciones del sur de Sudamérica mediante funciones discriminantes para el fémur. Revista Argentina de Antropología Biológica 10: 55-70.

Black, T.K. 1978. A new method for assessing the sex of fragmentary skeletal remains: femoral shaft circumference. American Journal of Physical Anthropology 48:227-231.

Buikstra, J.E. y D. Ubelaker 1994. Standards for Data Collection from Human Skeletal Remains. Fayetteville, AR, Arkansas Archeological Survey.

Byers, S.N. 2002. Introduction to Forensic Anthropology. Allyn and Bacon, Boston, MA.

Byrd, J.E. 2008. Models and Methods for Osteometric Sorting. En Recovery, Analysis, and Identification of Commingled Human Remains, editado por A. J. Bradley y J.E. Byrd, pp.199-220. Humana Press, New York.

Cameron, N. 2002. Human Growth and Development. Academic Press, California.

Chiavazza, H. 2005. Los Templos Coloniales como Estructuras Funerarias Arqueología en la Iglesia Jesuita de Mendoza. British Archaeological Reports International Series 1388, Londres.

Chiavazza, H. 2008. Bases teóricas para el análisis arqueológico de la espacialidad religiosa y los procesos de transformación cultural en la ciudad de Mendoza durante la colonia. Revista de Arqueología Americana 25:225-244

Chiavazza, H., D. Mansegosa, A. Gámez Mendoza y P.S. Giannotti 2015. Funebria católica y estimaciones del sexo y de la edad en entierros de una ciudad americana colonial (Mendoza, Argentina, siglos XVII-XIX) Revista de Arqueología Histórica Argentina y Latinoamericana 9:35-70.

Comadrán Ruiz, J. 1969. Evolución Demográfica Argentina durante el Período Hispano (1535-1810). EUDEBA, Buenos Aires.

Cowal, L.S. y R.F. Pastor 2008. Dimensional variation in the proxima ulna: evaluation of a metric method for sex assessment. American Journal of Physical Anthropology 135:469-478.

Di Bennardo, R. y J.V. Taylor 1982. Classification and misclassification in sexing the black femur by discriminant function analysis. American Journal of Physical Anthropology 58:145-151.

Frayer, D. y M. Wolpoff 1985. Sexual dimorphism. Annual Review of Anthropology 14:429-473.

Giannotti, S. 2016. Marcadores de estrés ocupacional en poblaciones históricas del norte de Mendoza (s. XVI-XIX): primeros resultados exploratorios. Comechingonia Revista de Arqueología 20:81-100.

Guimarey, L.M., F.R. Carnese y H.M. Pucciarelli 1996. La influencia ambiental en el crecimiento humano. Ciencia Hoy 5:41-47.

Gonzales-Reimers, E., J. Velasco-Vazquez, M. Arnay de la Rosa y F. Santolaria-Fernandez 2000. Sex determination by discriminant function analysis of the right tibia in the prehistoric population of the Canary Islands. Forensic Sciencie International 108:165-172.

Hair, J.,A. Rolph, T. Ronald y W.C. Black 1999. Analisis Multivariante. Pearson Educación, Madrid.

İsçan, M. Y., M. Yoshino y S. Kato 1994. Sex determination from the tibia. Journal Forensic Science 39: 785-792.
İşcan M.Y. y P. Miller-Shaivitz 1984. Discriminant function sexing of the tibia. Journal Forensic Science 29:1087-1093.

İşcan, M.Y., S.R. Loth, C.A. King, D. Shihai y M. Yoshino 1998. Sexual dimorphism in the humerus: A comparative analysis of Chinese, Japanese and Thais. Forensic Science International 98:1729.

King, Ch.A., Y.M. İşcan y S.R. Loth 1998. Metric and comparative analysis of sexual dimorphism in the Thai femur. Journal Forensic Science 43:954-958.

Leigh, S.R. 1992. Patterns of variation in the ontogeny of primate body size dimorphism. Journal of Human Evolution 23:27-50.

Lieberman, L.S. 1982. Normal and abnormal dimorphic patterns of growth and development. En Sexual Dimorphism in Homo Sapiens, editado por H. Roberta, pp. 263-312. Praeguer, New York.

Loth, S.R., y M. Henneberg 2001. Sexually dimorphic mandibular morphology in the first few years of life. American Journal of Physical Anthropology 115:179-187.

Mac Laughlin, S.M. y M.F. Bruce 1985. A simple univariate technique for determining sex from fragmentary femora: Its application to a Scottish short cist population. American Journal of Physical Anthropology 67:413-417.

Mall, G., M. Graw, K. Gehring y M. Hubig 2000. Determination of sex from femora. Forensic Science Inernational 113:315-321.

Mansegosa, D.A. 2010. Estudios bioarqueológicos en un templo colonial de Mendoza: La Caridad. En Actas del XVII Congreso Nacional de Arqueología Argentina. Mendoza.

Mansegosa, D.A. 2015a. Patrones de variación morfológica en poblaciones históricas del norte mendocino: aportes para el estudio del proceso de conquista y consolidación colonial. Tesis doctoral inédita. Universidad Nacional de La Plata.

Mansegosa, D.A. 2015b.Variación morfológica postcraneal en poblaciones históricas del norte de Mendoza: análisis de la relación entre la estatura y el índice de platimería. En Libro de resúmenes de las XXIV Jornadas de Investigación y VI Jornadas de Posgrado, pp. 45. Universidad Nacional de Cuyo, Mendoza.

Mansegosa, D.A. 2016. Estudios sobre salud y enfermedad en poblaciones históricas urbanas de Mendoza (Argentina). Nuevos aportes para el estudio bioarqueológico de La Caridad. Comechingonia virtual Revista de Arqueología 20:111-142.

Mansegosa, D.A., y H. Chiavazza 2010. Consecuencias del proceso de colonización en la salud de la población urbana de Mendoza (Argentina): un estudio desde evidencias paleopatológicas (S. XVIIIXIX). Revista Española de Paleopatología 8:1-18.

Mazza, B. y M. Béguelin 2013. Determinación sexual de los entierros secundarios del sitio arqueológico Cerro Lutz mediante funciones discriminantes de huesos largos. Cuadernos del Instituto Nacional de Antropología y Pensamiento Latinoamericano. Edición especial: I Congreso Internacional de la Cuenca del Plata 1:121-134.

Meindl, R.S., C.O. Lovejoy, R.P. Mensforth y L. Don Carlos 1985. Accuracy and direction of error in the sexing of the skeleton: implications for paleodemography. American Journal of Physical Anthropology 68:79-85.

Oyhenart, E.E., M.F. Cesani Rossi y H.M. Pucciarelli 1999. Influencia del retardo del crecimiento intrauterino sobre la diferenciación craneana postnatal. Revista Argentina de Antropología Biológica $2: 135-150$

Pomeroy, E. y S.R. Zakrzewski 2009. Sexual dimorphism in diaphyseal cross-sectional shape in the medieval Muslim population of Écija, Spain, and Anglo-Saxon Great Chesterford, UK. International Journal of Osteoarchaeology 19:50-65. 
Prieto, M.R. 2000 [1983]. Formación y consolidación de una sociedad en un área marginal del Reino de Chile: la Provincia de Cuyo en el siglo XVII. Anales del Instituto de Arqueología y Etnología 52-53: 18-366.

Pucciarelli, H.M., F.R. Carnese, L.V. Pinotti, L.M. Guimarey y A.S. Goicochea 1993. Sexual dimorphism in schoolchildren of the Villa IAPI neighborhood (Quilmes, Buenos Aires, Argentina). American Journal of Physical Anthropology 92:165-172.

Purkait, R. y Ch. Heeresh 2004. A study of sexual variation in Indian femur. Forensic Science International 146:25-33.

Robinson, M.S. y M.A. Bidmos 2009. The skull and humerus in the determination of sex: reliability of discriminant function equations. Forensic Science International 186:86.e1-5.

Ruff, Ch.B. 1994 Morphological adaptation to climate in modern and fossil hominids. Yearbook of Physical Anthropology 37:65-107.

Sakaue, K. 2004. Sexual determination of long bones in recent Japanese. Anthropological Science 112:75-81.

Schafer, J. y J. Graham 2002. Missing data: Our view of the state of the art. Psychological Methods 7:147-177.

Schafer, J. y M.K. Olsen 1998. Multiple imputation for multivariate missing-data problems: a data analyst's perspective. Multivariate Behavioral Research 33:545-571.

Šlaus, M. 1997. Discriminant function sexing of fragmentary and complete femora from medieval sites in continental Croatia. Opuscula Archaeologica 21: 167-175.

Šlaus, M., D. Strinović, J. Škavić y V. Petrovečki 2003. Discriminant function sexing of fragmentary and complete femora: standards for contemporary Croatia. Journal Forensic Science 48:509-512.

Šlaus, M. y Ž. Tomičić 2005. Discriminant function sexing of fragmentary and complete tibiae from medieval Croatian sites. Forensic Science International 147:147-152.

Steyn, M. y M. Y. İşcan 1997. Sex determination from the femur and tibia in South African whites. Forensic Science International 90:111-119.

Steyn, M. y M. Y. İşcan 1999. Osteometric variation in the humerus: sexual dimorphism in South Africans. Forensic Science International 106: 77-85.

Trancho, G., B. Robledo, I. Lopez-Bueis y A. Sanchez 1997. Sexual determination of femur using discriminant function analysis of a Spanish population of known sex and age. Journal Forensic Science 42:181-185.

Ubelaker, D.H. 1974. Reconstruction of Demographic Profiles from Ossuary Skeletal Samples: A Case Study from the Tidewater Potomac. Smithsonian Contributions to Anthropology 18, Washington.

Waghmare, J., P. Deshmukh y P. Waghmare 2012. Determination of sex from the shaft and tuberosity of radius a multivariate discriminant function analysis. Biomedical Research 23:115118.

White, T.D. y P.A. Folkens 2005. The Human Bone Manual. Elsevier Academic Press, San Diego.

Zuluaga, R.M. 1970. La trata de negros en la región cuyana durante el siglo XVII. Revista de la Junta de Estudios Históricos de Mendoza, Segunda Época 6(1). 